\title{
ACONSELHAMENTO NUTRICIONAL OFERECIDO A CRIANÇAS E ADOLESCENTES COM DEFICIÊNCIA
}

\author{
Nutritional counseling offered to children and adolescents \\ with disabilities
}
Consejo nutricional ofrecido a niños y adolescentes con discapacidad

Artigo Original

\section{RESUMO}

Objetivo: Avaliar o aconselhamento nutricional oferecido a crianças e adolescentes com deficiência no município de Santa Cruz-RN, Brasil. Métodos: Estudo observacional, transversal, de base populacional, desenvolvido de maio a dezembro de 2013, com 102 crianças e adolescentes atendidos em um Centro de Reabilitação Infantil. Procedeu-se avaliação socioeconômica, demográfica, de saúde e de estilo de vida, assim como da assistência nutricional recebida (se já haviam recebido orientação nutricional de algum profissional da saúde em relação à alimentação e nutrição, com que frequência ocorria e qual o profissional a havia realizado). Resultados: Somente $37 \%(n=37)$ dos responsáveis já haviam recebido alguma orientação nutricional. Com relação à frequência do aconselhamento, somente $11,8 \%$ $(n=4)$ dos jovens as recebeu 7 ou mais vezes/ano; 23,5\% $(n=8)$ as recebeu de 2 a 6 vezes/ ano; e a maioria $(64,7 \% ; n=22)$ as recebeu 1 vez/ano ou menos. O tempo de duração dessas orientações foi curto: a maioria $(64,7 \% ; n=22)$ recebeu apenas uma orientação durante todo o período em que foi acompanhado pela instituição, e menos de $15 \%(\mathrm{n}=5)$ obteve aconselhamento por mais de seis meses seguidos. Para a maioria dos jovens $(73,5 \%$; $=25)$, a quantidade de tempo recebendo orientações nutricionais não chegou à metade do período de acompanhamento. A orientação nutricional era realizada majoritariamente pelo nutricionista (89,2\%; n=33). Conclusão: Houve baixa frequência de aconselhamento nutricional específico para as deficiências apresentadas pelos participantes, e quando tal atividade foi desenvolvida, deu-se de forma irregular durante o acompanhamento no serviço, por período de tempo curto, e de maneira desarticulada do acompanhamento pela equipe multiprofissional.

Descritores: Criança; Adolescente; Pessoas com Deficiência; Aconselhamento; Nutrição de Grupos de Risco.

\section{ABSTRACT}

Objective: To evaluate the nutritional counseling offered to children and adolescents with disabilities in the municipality of Santa Cruz, RN, Brazil. Methods: Observational, crosssectional, population-based study, developed from May to December 2013, with 102 children and adolescents attending a Children's Rehabilitation Center. We conducted socioeconomic, demographic, health and lifestyle assessments, and evaluated the received nutritional assistance (whether they had received nutritional counseling from any health professional in relation to food and nutrition, how often it occurred and which professional conducted such counseling). Results: Only 37\% ( $n=37)$ of parents had received some nutritional guidance. Regarding the frequency of counseling, only $11.8 \%(n=4)$ of the youngsters received it 7 or more times/year; $23.5 \%(n=8)$ received it 2 to 6 times/year; and most $(64.7 \%, n=22)$ received it 1 timelyear or less. The length of the nutritional counseling was short: the majority $(64.7 \%, n=22)$ received only one guidance session throughout the whole period attending the institution, and less than $15 \%(n=5)$ received counseling for more than six consecutive months. For the majority $(73.5 \%, n=25)$, the amount of time receiving nutritional guidance has not reached half of the monitoring period. Nutritional counseling was carried out mainly by nutritionists $(89.2 \%, n=33)$. Conclusion: There was a low frequency of nutritional counseling directed specifically to the disabilities presented by the participants. When such activity was conducted, it occurred irregularly during the participant's follow-up, only

1) Universidade Federal do Rio Grande do Norte - UFRN - Natal (RN) - Brasil 
during a short period of time, and lacking connection with the monitoring by the multidisciplinary team.

Descriptors: Child, Adolescent; Disabled Persons; Counseling, Risk Groups Nutrition.

\section{RESUMEN}

Objetivo: Evaluar el consejo nutricional ofrecido a niños $y$ adolescentes con discapacidad del municipio de Santa Cruz-RN, Brasil. Métodos: Estudio observacional, transversal y de base poblacional desarrollado entre mayo y diciembre de 2013 con 102 niños y adolescentes asistidos en un Centro de Rehabilitación Infantil. Se hizo una evaluación socioeconómica, demográfica, de la salud y del estilo de vida así como de la atención nutricional recibida (si ya habian recibido orientación nutricional de algún profesional de la salud respecto la alimentación y la nutrición, con qué frecuencia se daba y cual profesional la había realizado). Resultados: Solamente el 37\% $(n=37)$ de los responsables ya habian recibido alguna orientación nutricional. Respecto la frecuencia del consejo solamente el 11,8\% $(n=4)$ de los jóvenes las recibió 7 o más veces al año; el 23,5\% $(n=8)$ las recibió entre 2 y 6 veces al año; y la mayoría $(64,7 \% ; n=22)$ la recibió 1 vez al año o menos. El tiempo de duración de estas orientaciones fue corto: la mayoría $(64,7 \% ; n=22)$ recibió solamente una orientación durante todo el periodo en el que fue acompañado por la institución y menos del 15\% $(n=5)$ recibió consejo más de seis meses. Para la mayoría de los jóvenes $(73,5 \% ; n=25)$ la cantidad de tiempo recibiendo las orientaciones nutricionales no alcanzó la mitad del periodo de consejo. La orientación nutricional fue realizada en la mayor parte de las veces por el nutricionista (89,2\%; n=33). Conclusión: Hubo baja frecuencia de consejo nutricional especifico para las deficiencias presentadas por los participantes y cuando la actividad fue desarrollada la misma se dio de manera irregular durante el seguimiento del servicio, en corto periodo de tiempo y de manera desarticulada del seguimiento del equipo multiprofesional.

Descriptores: Niño; Adolescente; Personas con Discapacidad; Consejo; Nutrición de Grupos Vulnerables.

\section{INTRODUÇÃO}

Pessoas com deficiência apresentam maior vulnerabilidade nutricional devido a alterações metabólicas e fisiopatológicas inerentes aos diferentes tipos de deficiência, predispondo a doenças crônicas não transmissíveis e outras comorbidades $^{(1)}$. Frequentemente, observa-se aumento da gordura corporal, obesidade, doenças cardiovasculares, osteoporose, alteração do metabolismo dos carboidratos, proteínas e lipídeos, cálculo renal, diabetes, hipertensão arterial, dislipidemia e síndrome metabólica ${ }^{(1-3)}$.

A deficiência também pode alterar o consumo alimentar e afetar a ingestão de energia e nutrientes ${ }^{(2-6)}$. Pessoas com dificuldade motora básica podem ter a função motora oral afetada ou ter dificuldade no controle dos movimentos da boca, cabeça, tronco e/ou membros, levando a desordens da mastigação, deglutição e/ou disfagia que podem resultar em desnutrição, desidratação, aspiração e pneumonia ${ }^{(7,8)}$. Em outros casos, há a necessidade de aporte calórico maior, pois gastam mais energia na execução dos movimentos ${ }^{(9)}$.

Além disso, distúrbios comportamentais presentes em determinadas deficiências (p. ex., recusa em comer, seletividade dos alimentos) e fatores extrínsecos ao próprio indivíduo (p. ex., dependência de terceiros para realizar as refeições, falta de autonomia na escolha da alimentação, falta de utensílios adaptados, dificuldade econômica, isolamento social) também podem potencializar a vulnerabilidade nutricional da pessoa com deficiência ${ }^{(10)}$.

Percebe-se, assim, que os cuidados com a alimentação e nutrição das pessoas com deficiência devem ser redobrados, fazendo-se imperativo o adequado aconselhamento nutricional voltado a essa população nos diferentes ciclos da vida ${ }^{(11)}$. Tal como qualquer outro cidadão, a pessoa com deficiência tem direito a serviços de promoção, prevenção, reabilitação e diagnósticos específicos nas unidades de saúde credenciadas pelo Sistema Único de Saúde ${ }^{(12,13)}$, de forma a obter cuidado integral e assistência multiprofissional, sob a lógica interdisciplinar ${ }^{(13)}$. Tais direitos fundamentais são resguardados pela Política Nacional de Saúde da Pessoa com Deficiência ${ }^{(14)}$, e desde a instituição da Rede de Cuidados à Pessoa com Deficiência ${ }^{(13)}$ pelo Ministério da Saúde, no ano de 2012, estão sendo cada vez mais assegurados a essa população.

$\mathrm{Na}$ prática, todavia, sabe-se que o aconselhamento nutricional das pessoas com deficiência ainda é bastante incipiente em todo o mundo. No Brasil, onde cerca de $24 \%$ da população apresenta algum tipo de deficiência ${ }^{(15)}$, existem poucos estudos investigando essa temática, particularmente em estados no Nordeste e regiões do interior desses estados, onde a atenção à pessoa com deficiência pode estar prejudicada. Nesse sentido, o presente artigo visa preencher essa lacuna do conhecimento, ao avaliar o aconselhamento nutricional oferecido a crianças e adolescentes com deficiência no município de Santa Cruz-RN, Brasil.

\section{MÉTODOS}

Trata-se de um estudo observacional, transversal, de natureza descritiva, realizado dentro de uma abordagem quantitativa. Participaram deste estudo crianças e adolescentes atendidos no Centro de Reabilitação Infantil (CRI) Monsenhor Raimundo Gomes Barbosa, localizado no município de Santa Cruz, interior do Rio Grande do Norte. A entidade acompanha jovens com diferentes tipos 
de deficiência e necessidades especiais, tais como paralisia cerebral, deficiência mental, física, auditiva, visual, distúrbio de comportamento, distúrbio de aprendizagem, imaturidade cerebral, distúrbio da fala, distúrbio neuropsicomotor e autismo.

O município de Santa Cruz-RN está situado a 111 quilômetros da capital do estado e figura como o município mais importante da microrregião Borborema Potiguar, sendo referência para essa região no interior do estado quanto à utilização dos seus serviços de saúde. Nesse contexto, o CRI de Santa Cruz é uma unidade de referência não somente para os moradores do município, mas também de cidades vizinhas, e o acompanhamento é realizado por equipe multiprofissional composta por médico, fisioterapeuta, fonoaudiólogo, nutricionista, terapeuta ocupacional e psicólogo.

A população do estudo consistiu na totalidade das crianças e adolescentes entre 0 e 19 anos de idade, de ambos os sexos, atendidos no CRI de Santa Cruz no ano de 2013 $(n=169)$. Visando-se avaliar a totalidade dessa população, invitaram-se todos os jovens elegíveis a participar. Os critérios de elegibilidade foram: ser acompanhado no CRI no momento do estudo, e o responsável aceitar a participação. Os critérios de exclusão foram: a presença de enfermidades ou agravos à saúde que impedissem a coleta de dados durante o período definido para a coleta de dados em campo (ex. internação hospitalar), ou o abandono do acompanhamento no CRI.

Dentre os 164 jovens acompanhados no CRI no momento do início da coleta de dados, 97 participaram (1 recusou; 7 abandonaram o CRI antes que os pesquisadores conseguissem convidá-los a participar; 1 aceitou participar, mas abandonou o CRI antes de ser avaliado; 58 não conseguiram ser convidados a participar pelos pesquisadores, não foram ao CRI durante todo o período de coleta de dados ou mudaram de endereço e/ou telefone). Outros 5 jovens admitidos no CRI durante a coleta de dados eram elegíveis e foram incluídos no estudo. Portanto, participaram do estudo um total de 102 crianças e adolescentes deficientes.

Realizou-se a coleta dos dados nas instalações do CRI, no período de maio a dezembro de 2013, mediante agendamento para o dia de acompanhamento habitual, nos horários de intervalo das consultas com os profissionais. Procedeu-se à entrevista contendo questões acerca da caracterização socioeconômica, demográfica, de saúde e de estilo de vida da criança ou adolescente, assim como da assistência nutricional recebida. Questionou-se se já haviam recebido orientação nutricional de algum profissional da saúde em relação à alimentação e nutrição, com que frequência ocorria e qual o profissional a havia realizado. Coletaram-se informações acerca da deficiência, sendo sua classificação feita com base nos Decretos n 3.298 , de 20 de dezembro de 1999 , e n ${ }^{\circ} 5.296$, de 2 de dezembro de 2004, e seu nível de gravidade definido segundo critérios adotados pelo Instituto Brasileiro de Geografia e Estatística ${ }^{(15)}$ no Censo Demográfico 2010. Quando o responsável não sabia ou não conseguia descrever adequadamente a deficiência da criança/adolescente (classificação, características etc.), tais informações foram coletadas nos prontuários da CRI, a fim de garantir a completude dos dados.

Devido à limitação do tempo disponível para a coleta dos dados de alguns participantes (residiam em outros municípios e dependiam do horário estabelecido pelo transporte da Secretaria Municipal de Saúde para a locomoção residência-CRI-residência), houve incompletude dos dados para algumas das variáveis investigadas, tais como número de moradores no domicílio $(\mathrm{n}=1)$, água encanada $(\mathrm{n}=1)$ e filtrada $(\mathrm{n}=1)$ no domicílio, e recebimento de aconselhamento nutricional específico para a deficiência apresentada $(n=3)$. Para outras variáveis, alguns participantes não souberam ou não quiseram responder ( $\mathrm{p}$. ex. cor da pele $(\mathrm{n}=15)$, renda familiar $(\mathrm{n}=16)$, frequência $(n=3)$ e duração $(n=3)$ do aconselhamento nutricional recebido), contribuindo também para a incompletude dos questionários e das variáveis construídas a partir dessas informações.

O estudo foi aprovado pelo Comitê de Ética em Pesquisa da Universidade Federal do Rio Grande do Norte (parecer $\mathrm{n}^{\circ}$ 487.774, protocolo CAAE $\mathrm{n}^{\circ}$ 12802813.7.0000.5537) e teve sua realização autorizada pelos dirigentes do CRI. Todos os procedimentos só foram efetuados após esclarecimento dos responsáveis pelos jovens quanto aos objetivos e procedimentos do estudo, e mediante assinatura do Termo de Consentimento Livre e Esclarecido, de acordo com a Resolução ${ }^{\circ}$ 466, de 12 de dezembro de 2012, do Conselho Nacional de Saúde.

As análises estatísticas foram realizadas utilizando o pacote estatístico SPSS versão 20.0, sendo considerado o valor de $\mathrm{p}<0,05$ para significância estatística. Inicialmente, descreveram-se as características demográficas, socioeconômicas e de saúde dos jovens, apresentandose a distribuição (absoluta e percentuais) das variáveis. Posteriormente, procedeu-se à análise bivariada para investigar a relação entre o tipo de deficiência e o aconselhamento nutricional oferecido aos participantes, com base no teste Qui-quadrado.

\section{RESULTADOS}

Dentre os 102 avaliados, a maior parte era do sexo masculino ( $\mathrm{n}=55 ; 53,9 \%)$ e tinha entre 5 e 9 anos de idade $(\mathrm{n}=57 ; 55,9 \%)$. Os deficientes, em sua maioria, tinham como chefe da família o pai ou a mãe ( $\mathrm{n}=94 ; 92,2 \%)$, os quais, em sua maioria, estudaram até o ensino fundamental $(n=57$; 
$55,9 \%)$. Os cuidados diários com deficiente também eram prestados geralmente pelo pai ou pela mãe $(n=91 ; 89,2 \%)$. Quase $83 \%$ das famílias $(\mathrm{n}=71)$ tinham renda familiar per capita inferior a meio salário mínimo, e em cerca de $69 \%$ dos lares $(\mathrm{n}=70)$ residiam mais de quatro pessoas.
Esgotamento sanitário para a rede pública e água encanada estavam presentes na maioria dos domicílios $(n=79 ; 77,5 \%$ e $\mathrm{n}=86 ; 85,1 \%$ respectivamente), mas água filtrada só era bebida em $50,5 \%$ das residências $(\mathrm{n}=51)$ (Tabela I).

\begin{tabular}{|c|c|c|}
\hline Variáveis & $\mathbf{n}$ & $\%$ \\
\hline Sexo & 102 & \\
\hline Masculino & 55 & 53,9 \\
\hline Feminino & 47 & 46,1 \\
\hline Faixa etária & 102 & \\
\hline 0 a 4 anos & 15 & 14,7 \\
\hline 5 a 9 anos & 57 & 55,9 \\
\hline 10 a 19 anos & 30 & 29,4 \\
\hline Cor da pele & 87 & \\
\hline Branca & 46 & 52,9 \\
\hline Preta / Parda & 41 & 47,1 \\
\hline Amarela / Indígena & 0 & 0,0 \\
\hline Chefe da família & 102 & \\
\hline Pai/Mãe & 94 & 92,2 \\
\hline Avô/Avó & 5 & 5,9 \\
\hline Outro & 2 & 2,0 \\
\hline Escolaridade do chefe da família & 102 & \\
\hline Analfabeto & 18 & 17,6 \\
\hline Sabe ler e escrever / ensino fundamental & 57 & 55,9 \\
\hline Ensino médio / ensino superior & 27 & 26,5 \\
\hline Cuidador principal do deficiente & 102 & \\
\hline Pai/Mãe & 91 & 89,2 \\
\hline Avô/Avó & 7 & 6,9 \\
\hline Outro parente & 4 & 3,9 \\
\hline Escolaridade do cuidador principal do deficiente & 102 & \\
\hline Analfabeto & 6 & 5,9 \\
\hline Sabe ler e escrever / ensino fundamental & 65 & 63,7 \\
\hline Ensino médio / ensino superior & 31 & 30,4 \\
\hline Renda familiar per capita* & 86 & \\
\hline$<0,25$ salários mínimos & 34 & 39,5 \\
\hline 0,25 a 0,50 salários mínimos & 37 & 43,0 \\
\hline >0,50 salários mínimos & 15 & 17,4 \\
\hline Número de moradores no domicílio & 101 & \\
\hline Até 4 pessoas & 31 & 30,7 \\
\hline$>4$ pessoas & 70 & 69,3 \\
\hline Água encanada no domicílio & 101 & \\
\hline Sim & 86 & 85,1 \\
\hline Não & 15 & 14,9 \\
\hline Água filtrada no domicílio & 101 & \\
\hline Sim & 51 & 50,5 \\
\hline Não & 50 & 49,5 \\
\hline Esgotamento sanitário & 102 & \\
\hline Rede pública & 79 & 77,5 \\
\hline Fossa & 17 & 16,7 \\
\hline Outra forma & 6 & 5,8 \\
\hline
\end{tabular}

* Para este cálculo, considerou-se como referência o valor do salário mínimo nacional de $\mathrm{R} \$ 678,00$, vigente no ano de 2013. 
As deficiências mais frequentes foram a mental $(n=76$; $74,5 \%)$ e a física $(\mathrm{n}=11 ; 10,8 \%)$. Cerca de $25 \%(\mathrm{n}=25)$ apresentava adversidades ao se alimentar, tais como dificuldade na mastigação e deglutição $(n=8)$, dificuldade no controle da cabeça, tronco e/ou membros $(n=11)$ ou dificuldades múltiplas $(\mathrm{n}=6)$. A capacidade de alimentar-se sozinho era limitada em 18\% $(n=18)$ dos jovens, dos quais metade ( $n=9)$ não conseguia realizar essa tarefa sozinha, mesmo com o auxílio de pessoas e/ou utensílios adaptados (Tabela II).

Ao avaliar o aconselhamento nutricional oferecido às crianças e adolescentes com deficiência, verificouse que somente $37 \%(\mathrm{n}=37)$ dos responsáveis já haviam recebido alguma orientação nutricional desde o início do acompanhamento da criança/adolescente no CRI, sendo mais frequente entre aqueles com deficiências múltiplas ou deficiência física. O profissional que comumente realizava a orientação sobre alimentação e nutrição era o nutricionista
$(89,2 \% ; n=33)$, seguido do médico ou enfermeiro $(5,4 \%$; $\mathrm{n}=2$ ) (Tabela III).

Com relação à frequência do aconselhamento, isto é, à regularidade no recebimento dessas orientações nutricionais específicas para a deficiência, somente $11,8 \%$ dos jovens $(\mathrm{n}=4)$ as recebeu 7 ou mais vezes/ano; $23,5 \%(\mathrm{n}=8)$ as recebeu de 2 a 6 vezes/ano; e a maioria $(\mathrm{n}=22 ; 64,7 \%)$ as recebeu 1 vez/ano ou menos. O tempo de duração dessas orientações, que reflete a continuidade do aconselhamento nutricional, também foi curto: a maioria $(n=22 ; 64,7 \%)$ recebeu apenas uma orientação durante todo o período em que foi acompanhado pela instituição, e menos de $15 \%(\mathrm{n}=5)$ obteve aconselhamento por mais de seis meses seguidos. Como consequência, verificou-se que, para a maioria dos jovens $(\mathrm{n}=25 ; 73,5 \%)$, a quantidade de tempo recebendo orientações nutricionais não chegou à metade do período de acompanhamento no CRI (Tabela III), que, em média, foi de 32,2 meses para a população estudada.

Tabela II - Características de saúde das crianças e adolescentes com deficiência acompanhados no Centro de Reabilitação Infantil. Santa Cruz-RN, 2013.

\begin{tabular}{lcc}
\hline Variáveis & $\mathbf{n}$ & $\mathbf{\%}$ \\
\hline Tipo de deficiência & $\mathbf{1 0 2}$ & 1,0 \\
Visual & 1 & 2,9 \\
Auditiva & 6 & 10,8 \\
Física & 11 & 74,5 \\
Mental & 76 & 7,8 \\
Múltipla & 8 & $\mathbf{1 0 2}$ \\
Tempo de acompanhamento na instituição & 38 & 37,3 \\
Até 12 meses & 28 & 27,5 \\
13 a 36 meses & 36 & 35,3 \\
37 meses ou mais & $\mathbf{1 0 2}$ & 77 \\
Dificuldades ao alimentar-se & 77,5 \\
Nenhuma & 8 & 7,8 \\
Mastigação/deglutição & 11 & 10,8 \\
Controle dos movimentos de cabeça, tronco e/ou membros & 6 & 5,9 \\
Múltiplas dificuldades & $\mathbf{1 0 2}$ & 82,2 \\
Capacidade de alimentar-se sozinho & 84 & 8,8 \\
Alimenta-se sozinho, sem auxílio de pessoas e/ou utensílios adaptados & 9 & 8,8 \\
Alimenta-se sozinho, mas necessita de auxílio de pessoas e/ou utensílios adaptados & 9 & \\
Não se alimenta sozinho, mesmo com auxílio de pessoas e/ou utensílios adaptados & & \\
\hline
\end{tabular}




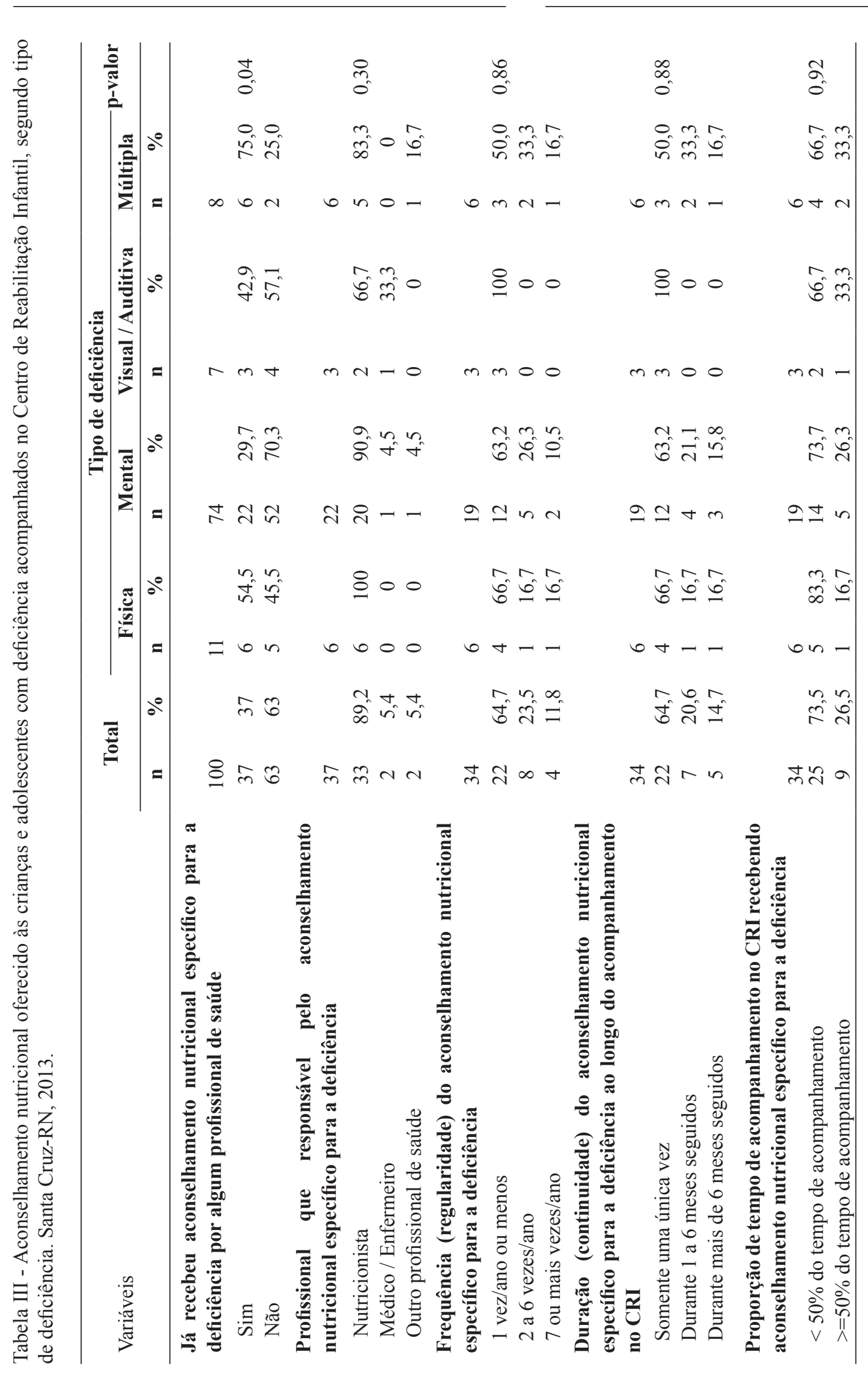




\section{DISCUSSÃO}

Este estudo verificou baixa frequência de aconselhamento nutricional específico para as deficiências apresentadas pelas crianças e adolescentes investigados. Tais ações, quando foram desenvolvidas, deram-se de forma irregular durante o acompanhamento do jovem no serviço, por período de tempo curto e de maneira desarticulada do acompanhamento pela equipe multiprofissional. Tal assistência vai em direção oposta àquela preconizada pela Rede de Cuidados à Pessoa com Deficiência no âmbito do Sistema Único de Saúde ${ }^{(13)}$, em que deve haver garantia de acesso e de qualidade dos serviços, com cuidado integral e assistência multiprofissional interdisciplinar.

Esse cenário torna-se ainda mais crítico ao considerar que os jovens arrolados no estudo são residentes de cidades do interior de um estado do Nordeste do país, têm baixo nível socioeconômico e dependem de cuidadores com baixa escolaridade, o que frequentemente resulta em limitado acesso a informações sobre o manejo nutricional adequado na deficiência e impossibilidade de realizar acompanhamento em serviços de saúde privados.

Evidencia-se, portanto, que, embora a temática da promoção da saúde e da integralidade do cuidado venha ganhando cada vez mais destaque no Brasil nos últimos anos, ainda não há grande visibilidade para as pessoas que possuem algum tipo de deficiência ${ }^{(16)}$.

O acesso a serviços de promoção da saúde são fundamentais na determinação da qualidade de vida e do estado de saúde de pessoas com deficiência ${ }^{(16,17)}$. A Política Nacional de Promoção da Saúde ${ }^{(18)}$ define a promoção da saúde como uma estratégia de "produção de saúde", ou seja, como um conjunto de ações articuladas desenvolvidas no sistema público de saúde do Brasil que possa contribuir para suprir as necessidades da sociedade no que se refere à saúde. Ao promover a saúde dessas pessoas, contribuise também para a prevenção de doenças secundárias, tais como: obesidade, hipertensão, diabetes, entre outras, que podem aparecer devido à deficiência e modo de vida ${ }^{(19)}$.

Nesse contexto, o aconselhamento nutricional específico para as necessidades particulares da pessoa com deficiência é um elemento de grande importância para garantir a manutenção da saúde e prevenir o aparecimento de distúrbios nutricionais. É necessária uma orientação nutricional, tanto individual como familiar, a fim de minimizar a monotonia alimentar e oferecer um cardápio equilibrado e variado para garantir um melhor estado de saúde $^{(10,20)}$. Embora ainda não haja consenso na literatura, estudos pontuais relatam o impacto positivo da intervenção nutricional e promoção da alimentação saudável em pessoas com deficiência, com evolução positiva nos parâmetros nutricionais ${ }^{(11,21,22)}$.
Em nosso país, pode-se notar a falta de serviços relacionados à promoção e prevenção da saúde para pessoas com deficiência ${ }^{(23,24)}$, além de dificuldades de acesso a serviços ambulatoriais de maneira geral( ${ }^{(25)}$, levando-as a procurar diretamente serviços especializados e hospitais para atendimento, o que sobrecarrega esses estabelecimentos e demonstra que os níveis primários de atendimento não estão acessíveis a essa população ${ }^{(25)}$. No presente estudo, mesmo com o acesso dos jovens com deficiência ao Centro de Reabilitação Infantil, não houve garantia de um atendimento que contemplasse todas as suas necessidades, particularmente a orientação nutricional ao longo do acompanhamento. A falta de profissionais capacitados e a escassez de sistemas de serviços ou rigor com políticas públicas destinadas a prestar apoio para as pessoas com deficiência constituem grandes barreiras ${ }^{(17)}$.

Considerando a importância do aconselhamento nutricional, a American Dietetic Association ${ }^{(4)}$ reforça que o nutricionista é essencial em serviços de saúde que atendem pessoas com necessidades especiais. Esses profissionais desempenham um papel fundamental na equipe de saúde, nas avaliações clínicas, bioquímicas, antropométricas, dietéticas, de habilidades de alimentação, bem como na compreensão dos fatores ambientais, sociais, econômicos e educacionais que afetam a terapêutica da pessoa com deficiência. Eles devem desenvolver ou adotar critérios e ferramentas de triagem, avaliação e monitoramento nutricional, e têm a responsabilidade de orientar familiares e cuidadores acerca da seleção e preparo de alimentos como parte da terapêutica.

Todavia, não somente o nutricionista, mas todos os membros da equipe multiprofissional devem estar envolvidos na terapêutica nutricional da pessoa com deficiência, inclusive no que diz respeito à orientação sobre utensílios adaptados para auxiliar a alimentação ou texturas e consistências seguras de alimentos para o consumo ${ }^{(4)}$. $\mathrm{O}$ fato de, neste estudo, as orientações nutricionais terem sido oferecidas majoritariamente pelo profissional nutricionista demonstra a falta de compromisso da equipe multiprofissional em relação à alimentação, a qual parece não estar sendo vista como um elemento importante do processo de reabilitação da pessoa com deficiência. Assim, torna-se necessário fortalecer, na prática desses profissionais, o conceito da integralidade do cuidado, a fim de atentarem para a dimensão da alimentação e sua relação com a qualidade de vida nessa população.

Nas ações de saúde voltadas para pessoas com deficiência, é imprescindível que se leve em conta um mosaico de diferentes necessidades e especificidades ${ }^{(23)}$, já que constituem um grupo heterogêneo, que reúne em uma mesma categoria indivíduos que podem ter diferentes condições motoras, sensoriais, intelectuais ou múltiplas. 
Neste estudo, contudo, verificou-se que o aconselhamento nutricional foi desigual entre os diferentes tipos de deficiência, o que possivelmente reflete o despreparo dos profissionais para orientar a alimentação mais adequada para determinadas deficiências, tais como a mental, em que menos de $30 \%$ dos participantes já havia recebido orientações nutricionais. Segundo a American Dietetic Association $^{(4)}$, é importante que existam programas de educação continuada interdisciplinares em nutrição voltados à pessoa com deficiência, para expandir o conhecimento de profissionais nessas áreas do conhecimento.

Reconhece-se que o aconselhamento nutricional não é tarefa fácil em pessoas com deficiência, pois embora haja vulnerabilidade nutricional nesta população, ainda não foram desenvolvidos, nem no Brasil nem em outros países, protocolos nutricionais padronizados ou recomendados oficialmente para diagnóstico, monitoramento e reabilitação nutricional de pessoas com diferentes tipos de deficiência ${ }^{(4)}$, tampouco recomendações acerca de como prevenir, inibir ou minimizar distúrbios nutricionais nesse grupo com vistas a melhorar sua qualidade de vida. Assim, as tomadas de decisões ainda são inespecíficas e baseadas nos parâmetros da população sem deficiência ${ }^{(1)}$, o que é inapropriado. Nesse sentido, a American Dietetic Association ${ }^{(4)}$ recomenda que os pesquisadores da área de nutrição se debrucem acerca dessa temática, de forma que sejam oferecidos subsídios aos profissionais de saúde para desenvolver as orientações nutricionais mais adequadas a cada deficiência.

Tal como observado neste estudo, é possível que em outros serviços de saúde o aconselhamento nutricional não esteja sendo adequadamente inserido no processo de reabilitação de indivíduos com deficiência assistidos, o que não atende aos princípios da universalidade, da acessibilidade, da continuidade do cuidado e da integralidade da atenção, conforme preconizado pelo Ministério da Saúde. Faz-se necessário que o nutricionista, assim como os outros profissionais de saúde da equipe multiprofissional, amplie o rol de ações de alimentação e nutrição voltadas a esse grupo, visando prevenir problemas nutricionais e aumentar sua qualidade de vida.

Recomenda-se também que estudos similares ao apresentado sejam desenvolvidos em outras localidades, a fim de se evidenciar o real cenário do aconselhamento nutricional oferecido a crianças e adolescentes com deficiência no Brasil. Tais informações serão de fundamental importância para identificar pontos frágeis na atenção nutricional prestada às pessoas com deficiência e definir as estratégias visando à superação de tais entraves nas ações de promoção, prevenção e recuperação da saúde e do adequado estado nutricional em pessoas com deficiência. Serão úteis também para subsidiar o planejamento e execução de ações factíveis e efetivas de alimentação e nutrição voltadas a esse grupo, a fim de prevenir e recuperar desvios nutricionais e agravos à saúde.

\section{CONCLUSÃO}

Houve baixa frequência de aconselhamento nutricional específico para as deficiências apresentadas pelos participantes, e quando tal atividade foi desenvolvida, deuse de forma irregular durante o acompanhamento no serviço, por período de tempo curto e de maneira desarticulada do acompanhamento pela equipe multiprofissional.

\section{REFERÊNCIAS}

1. Abreu T, Friedman R, Fayh APT. Aspectos fisiopatológicos e avaliação do estado nutricional de indivíduos com deficiências físicas. Rev HCPA. 2011;31(3):345-52.

2. Sabour H, Javidan AN, Ranjbarnovin N, Vafa MR, Khazaeipour Z, Ghaderi F, et al. Cardiometabolic risk factors in Iranians with spinal cord injury: analysis by injury-related variables. J Rehabil Res Dev. 2013;50(5):635-42.

3. Marín AS, Xandri JM. Nutritional status of intellectual disabled persons with Down syndrome. Nutr Hosp. 2011;26(5):1059-66.

4. American Dietetic Association. Position of the American Dietetic Association: providing nutrition services for people with developmental disabilities and special health care needs. J Am Diet Assoc. 2010;110(2):296-307.

5. Bertoli S, Battezzati A, Merati G, Margonato V, Maggioni M, Testolin G et al. Nutritional Status and dietary patterns in disabled people. Nutr Metab Cardiovasc Dis. 2006; 16:100-12.

6. Valter CA, Panziera C, Ribeiro JL, Sant'Anna MM, Fayh APT. Perfil antropométrico e consumo alimentar de indivíduos com deficiência praticantes de natação e futsal. EFDeportes [periódico na Internet]. 2010 [acesso em 2015 fev 10]; 15(150). Disponível em: http:// www.efdeportes.com/efd150/consumo-alimentar-deindividuos-com-deficiencia.htm

7. Okeke IB, Ojinnaka NC. Nutritional Status of Children with Cerebral Palsy in Enugu Nigeria. Eur J Sci Res. 2010;39(4):505-13.

8. Benfer KA, Weir KA, Bell KL, Ware RS, Davies PS, Boyd RN. Food and fluid texture consumption in a population-based cohort of preschool children with 
cerebral palsy: relationship to dietary intake. Dev Med Child Neurol. 2015 [Epub ahead of print]

9. Mohanty RK, Lenka P, Equebal A, Kumar A. Comparison of energy cost in transtibial amputees using "prosthesis" and "crutches without prosthesis" for walking activities. Ann Phys Rehabil Med. 2012;55(4):252-62.

10. Campos MA, Sousa R. Nutrição e Deficiência(s). Lisboa: Direção-Geral da Saúde; 2015.

11. Bertoli S, Spadafranca A, Merati G, Testolin G, Veicsteinas A, Battezzati A. Nutritional counselling in disabled people: effects on dietary patterns, body composition and cardiovascular risk factors. Eur J Phys Rehabil Med. 2008;449(2):149-58.

12. Ministério da Saúde (BR), Secretaria de Atenção à Saúde, Departamento de Ações Programáticas Estratégicas. A pessoa com deficiência e o Sistema Único de Saúde. $2^{\mathrm{a}}$ ed. Brasília: Ministério da Saúde; 2008.

13. Ministério da Saúde (BR). Portaria No 793 , de 24 de abril de 2012. Diário Oficial da União 25 abr 2012; 1:94-95.

14. Ministério da Saúde (BR), Secretaria de Atenção à Saúde. Política Nacional de Saúde da Pessoa com Deficiência. Brasília: Editora do Ministério da Saúde; 2010.

15. Instituto Brasileiro de Geografia e Estatística. Censo Demográfico 2010: características gerais da população, religião e pessoas com deficiência. Rio de Janeiro: IBGE; 2010.

16. Interdonato GC, Greguol M. Promoção da saúde de pessoas com deficiência: uma revisão sistemática. HU Rev. 2012;37(3):369-75.

17. Smith RD. Promoting the health of people with physical disabilities: a discussion of the financing and organization of public health services in Australia. Health Promot Internation. 2000;15(1):79-86.

18. Ministério da Saúde (BR), Secretaria de Atenção à Saúde. Política Nacional de Promoção da Saúde. Brasília: Ministério da Saúde; 2012.
19. Rimmer JH, Rowland JL, Yamaki K. Obesity and secondary conditions in adolescents with disabilities: addressing the needs of an underserved population. J Adolesc Health. 2007;41(3):224-9.

20. Lira MKA, Bion FM, Pessoa DCNP, Souza EF, Vasconcelos DAA. Perfil socioeconômico, estado nutricional e consumo alimentar de portadores de deficiência mental. Rev Bras Nutr Clin. 2010;25(3):21823.

21. Faria ER, Marinho MS, Abranches MV, Fonseca LA, Priore SE. Evolução e Impacto da Intervenção Nutricional em Portadores de Necessidades Especiais Assistidos pela APAE de Viçosa, MG. In: Anais do $2^{\circ}$ Congresso Brasileiro de Extensão Universitária, Belo Horizonte, 2004 Set 12 a 15 [acesso em 2015 Fev 05]. Disponível em: www.ufmg.br/congrext/Saude/ Saude86.pdf

22. Souza NS, Horsts RFML. Avaliação da Educação Nutricional para portadores de Síndrome de Down em Associações de Pais e Amigos dos Excepcionais (APAE's) do Vale do Aço, MG. Nutrir Gerais. 2011;5(9):770-82.

23. Bernardes LCG, Maior IMML, Spezia CH, Araújo TCCF. Pessoas com deficiência e políticas públicas no Brasil: reflexões bioéticas. Ciênc Saúde Coletiva 2009;14(1):31-8.

24. Castro SS, Lefèvre F, Lefèvre AMC, Cesar CLG. Acessibilidade aos serviços de saúde por pessoas com deficiência. Rev Saúde Pública. 2011;45(1):99-105.

25. Freire DB, Gigante LP, Béria JU, Palazzo LS, Figueiredo ACL, Raymann BCW. Acesso de pessoas deficientes auditivas a serviços de saúde em cidade do sul do Brasil. Cad Saúde Pública. 2009;25(4):889-97.

\section{Endereço para correspondência:}

Ursula Viana Bagni

Universidade Federal do Rio Grande do Norte

Departamento de Nutrição

Av. Senador Salgado Filho, 3000

Bairro: Lagoa Nova - Campus Universitário

CEP 59078-970 - Natal - RN - Brasil

E-mail: ursulaviana@gmail.com 